\title{
Research Paper \\ Comparison of Personality Correlates of Machiavellianism, Narcissism and Psychopathy (Dark Triad of Personality) in Three Factor Personality Model
}

\author{
Ali Mohammadzadeh ${ }^{1},{ }^{*}$ Ahmad Ashouri ${ }^{2}$
}

1. PhD in Psychology, Associate Professor, Department of Psychology, Payame Noor University, Tehran, Iran.

2. PhD in Clinical Psychology, Assistant Professor, Department of Clinical Psychology, School of Behavioral Sciences and Mental Health (Tehran Institute of Psychiatry), Iran University of Medical Sciences, Tehran, Iran.

\begin{tabular}{|c|c|}
\hline $\begin{array}{l}\text { Use your device to scan } \\
\text { and read the article online }\end{array}$ & \\
\hline 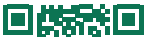 & \\
\hline riph & \\
\hline pitisy & 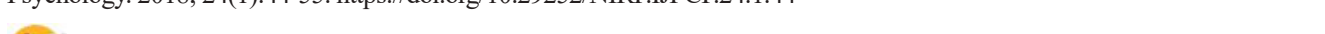 \\
\hline aprising & dol": https://doi.org/10.29252/NIRP.IJPCP.24.1.44 \\
\hline
\end{tabular}

Received: 09 Jan. 2017 Accepted: 30 Oct. 2017

Key words: Machiavellianism, Narcissism, Antisocial personality, Personality disorders

\section{A B S TRACT}

Objectives Machiavellianism, Narcissism and Psychopathy are often referred to as the dark triad of personality. This study explored the relationships of those personalities with three factor personality models.

Methods The current study was conducted in correlational descriptive research context. A group of 203 normal participants from Tabriz Payame noor university students answered to MACH-IV, MCMI-II and $E P Q-R$ personality questionnaires. Data analyzed using the stepwise multivariate regression analysis method.

Results According to three factors model of personality, Machiavellianism was correlated to neuroticism $(r=0.46, P<0.01)$ and psychoticism $(r=0.32, P<0.01)$, Narcissism was correlated to psychoticism $(r=0.17$, $P<0.01)$ and extraversion $(r=0.17, P<0.01)$, Psychopathy was correlated to psychoticism $(r=0.43, P<0.01)$ and neuroticism ( $r=0.43, \mathrm{P}<0.01)$.

Conclusion The dark dimension of personality can be described in terms of high psychoticism, according to three factor personality model.

\section{Extended Abstract}

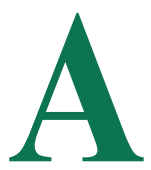

\section{Introduction}

mong socially aversive personality traits that pointed out by Kowalski [1], three have attracted the most empirical attention from the research context: Machiavellianism, Narcissism and Psychopathy. Despite their diverse routs, the personalities composing Dark Triad share a number of charectristics. All three entail asocially malevolent character with behavior tendencies toward self-promotion, emotional coldness, duplicity, and aggressiveness
[2]. Based on the literature, especially in non-clinical groups, evidence suggests overlapping between Machiavellianism, narcissistic and antisocial personality [2-5]. It seems that individuals with dark personality traits have traits that can be considered from the general traits of personality characteristics.

The measurement of the normal and abnormal dimensions of personality traits has a long history. One of these credible systems is based on the self-report belong to Eisenck's theory and his three-dimensional perspective of personality. These three dimensions of personality are: extraversion versus introversion, neu-

* Corresponding Author:

Ahmad Ashouri, PhD

Address: Department of Clinical Psychology, School of Behavioral Sciences and Mental Health (Tehran Institute of Psychiatry), Iran University of Medical Sciences, Tehran, Iran.

Tel: +98 (21) 66506853

E-mail: ahmad.ashouri@gmail.com 
roticism versus emotional stability, psychoticim versus impulse control [6].

To date, there has been afew research on the relationships between personality trait and the Dark Triad. In the few studies carried out on the basis of the five-factor model, Paulhus and Williams [2] as well as Jakobwitz and Egan [3] showed that agreeableness tend to have negatively higher correlations with Machiavellianism, narcissistic and antisocial personality. This study explored the relationships of those personalities with three factor personality models.

\section{Methods}

The study was a descriptive-analytical study. The study population consisted of all Tabriz Payame Noor University students in the academic year of 2014-15. Stratified random sampling was used to select the subjects.
Given the nature of research and considering the adequacy of subject numbers in the research, the sample size was set at 203 (Average age $=29.10$ years, $\mathrm{SD}=7.6$ ). Male mean age was 31.53, $\mathrm{SD}=7.63,(\mathrm{~N}=58)$. Female mean age was 28.17, $\mathrm{SD}=7.45(\mathrm{~N}=145)$. For data analysis, stepwise multiple regression analysis was performed using SPSS 21 software. Research tools included MACH-IV, MCMI-II and EPQ-R personality questionnaires.

\section{Results}

Pearson correlation coefficient was used in order to explore the relationship between the dimensions of personality traits and dark triad personality. These results are summarized in Table 1.

As the dark triad personality dimension appeared to vary by Eysenck personality traits, we used multiple regression analysis models. Tables 2, 3 and 4 show the results of the

Table 1. Correlations between dimensions of personality traits and dark triad personality

\begin{tabular}{|c|c|c|c|c|c|c|}
\hline Component & 1 & 2 & 3 & 4 & 5 & 6 \\
\hline Machiavellianism & 1 & $0.15^{*}$ & $0.37 * *$ & $0.46^{* *}$ & $0.32 * *$ & $0.23 * *$ \\
\hline Narcissism & & 1 & $0.43^{*}$ & 0.11 & $0.17^{*}$ & $0.17^{*}$ \\
\hline Psychopathy & & & 1 & $0.43^{* *}$ & $0.43 * *$ & 0.06 \\
\hline Neuroticism & & & & 1 & $0.41 * *$ & $-0.21 * *$ \\
\hline Psychoticism & & & & & 1 & 0.17 \\
\hline Extraversion & & & & & & 1 \\
\hline
\end{tabular}

Table 2. Regression analysis of Machiavellianism on three-factor personality model

\begin{tabular}{|c|c|c|c|c|c|c|c|c|c|}
\hline Step & Predictor Variable & $r$ & $\mathbf{R}^{2}$ & $\mathbf{F}$ & Sig. & B & b & $\mathbf{t}$ & Sig. \\
\hline First & Neuroticism & 0.46 & 0.21 & 55.32 & 0.001 & 0.63 & 0.46 & 7.43 & 0.001 \\
\hline \multirow{2}{*}{ Second } & Neuroticism & \multirow{2}{*}{0.48} & \multirow{2}{*}{0.23} & \multirow{2}{*}{31.05} & \multirow{2}{*}{0.001} & 0.54 & 0.39 & 5.88 & 0.001 \\
\hline & Psychoticism & & & & & 0.51 & 0.15 & 2.35 & 0.02 \\
\hline
\end{tabular}

Table 3. Regression analysis of Narcissism on three-factor personality model

\begin{tabular}{|c|c|c|c|c|c|c|c|c|c|}
\hline Step & Predictor Variable & $r$ & $\mathbf{R}^{2}$ & $\mathbf{F}$ & Sig. & B & b & $\mathbf{t}$ & Sig. \\
\hline First & Psychoticism & 0.17 & 0.03 & 5.97 & 0.001 & 0.28 & 0.17 & 2.44 & 0.001 \\
\hline \multirow[t]{2}{*}{ Second } & \multirow[t]{2}{*}{ Psychoticism extraversion } & \multirow[t]{2}{*}{0.27} & \multirow[t]{2}{*}{0.07} & \multirow[t]{2}{*}{7.51} & \multirow[t]{2}{*}{0.001} & 0.35 & 0.21 & 3.03 & 0.003 \\
\hline & & & & & & 0.21 & 0.21 & 2.97 & 0.003 \\
\hline
\end{tabular}


Table 4. Regression analysis of Psychopathy on three-factor personality model

\begin{tabular}{|c|c|c|c|c|c|c|c|c|c|}
\hline Step & Predictor Variable & $\mathbf{r}$ & $\mathbf{R}^{2}$ & $\mathbf{F}$ & Sig. & B & b & $\mathbf{t}$ & Sig. \\
\hline First & Psychoticism & 0.43 & 0.18 & 43.28 & 0.001 & 0.68 & 0.42 & 6.57 & 0.001 \\
\hline Second & Psychoticism Neuroticism & 0.51 & 0.26 & 33.61 & 0.001 & $\begin{array}{l}0.48 \\
0.20\end{array}$ & $\begin{array}{l}0.30 \\
0.30\end{array}$ & $\begin{array}{l}4.46 \\
4.44\end{array}$ & 0.001 \\
\hline
\end{tabular}

triple analysis results. According to Table 4, regression analysis results showed that in first step the entrance of Neuroticism can significantly explain $21 \%$ of Machiavellianism variances. In the second step, Psychoticism can raise this amount to $23 \%$.

According to Table 3, regression analysis results showed that in first step the entrance of Psychoticism can significantly explain $3 \%$ of narcissism variances. In the second step, extraversion can raise this amount to $7 \%$. According to Table 3, regression analysis results showed that in first step the entrance of Psychoticism can significantly explain $18 \%$ of Psychopathy variances. In the second step, extraversion can raise this amount to $26 \%$.

\section{Discussion}

The results of this study showed that, based on Eisenck's three-factor model: 1. The Machiavellianism personality has a positive relationship with neuroticism and Psychoticism. It seems that the important characteristics of Machiavellianism personality are high neuroticism, and this may explain some of their features, such as the overcoming of negative affect, alexithymic and anhedonic states, which are also seen in a wide range of neurotic disorders. This finding is consistent with the findings of McHoskey [7] Ali et al [8]. Characteristics such as the lack of emotional involvement with others and the emotional detachment of the Machiavellian personality, described by Wrightsman [9], are well suited to Eysenck's concept of psychoticism.

2. The Narcissitic personality has a positive relationship with psychoticism and extraversion. Psychoticism inlcude features such as emotional coldness, antisocial behavior, and disability in empathy with others and seems to be more closely associated with the characteristics of narcissistic personality. The positive relationship between narcissism and extraversion found in the present study is consistent with earlier studies which have suggested that high extraversion and low agreeableness are characteristic of narcissistic people [10-13].

3. The antisocial personality has a positive relationship with neuroticism and Psychoticism. The relationship be- tween antisocial personality and Eisench's Psychoticism was completely expected, because the Eysenck Psychoticism scale measures mainly antisocial tendencies [14]. Also, the tendency to experience negative emotions in a neuroticism can be explained by some antisocial personality syndromes. The findings of this section of the study are consistent with the results of previous studies [2-5]. In a summing up, the dark dimension of personality can be described in terms of high psychoticism, according to three factor personality model.

The present study was limited in two respects; one was that research tools were long-term and this issue may have affected the results by introducing fatigue in the participants. Second, the study was conducted in a non-clinical sample, since clinical patients with personality disorders were not present in this study, this limitation should be considered in generalizing the results, based on this, it is recommended that the results of this study be investigated in these groups.

\section{Ethical Considerations}

\section{Funding}

This research did not receive any specific grant from funding agencies in the public, commercial, or not-forprofit sectors.

\section{Conflict of Interest}

The authors declare no conflict of interest.

\section{Acknowledgements}

The students of Payame Noor University of Tabriz who helped us in this research, are appreciated. 


\title{
مقايسه همبستهاى شخصيتى صفات ماكياوليايى، خودشيفتكى وضداجتماعى (مثلث تاريكشخئميتى) در التَوى سهعاملى مهماي
}

\author{
على محمدزاده'، "احمد عاشورى"
}

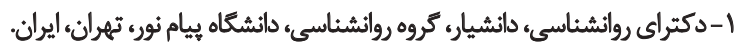

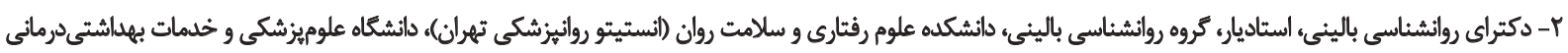
ايران، تهران، ايران.

\section{דكي4

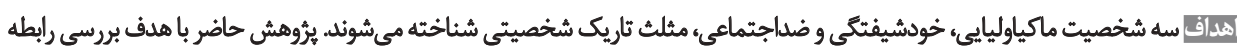

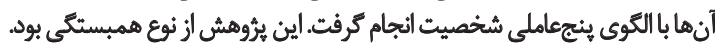

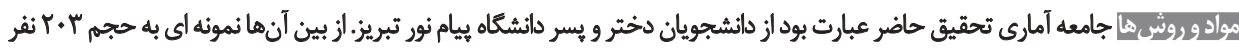

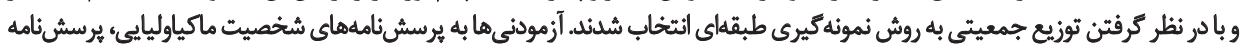

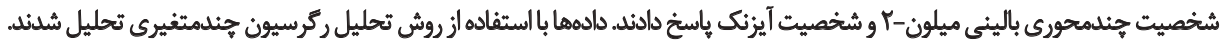

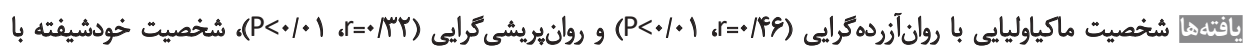

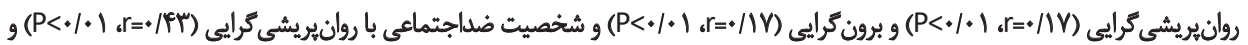

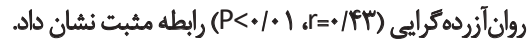

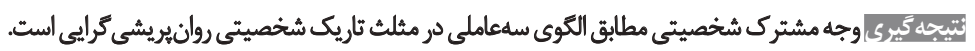

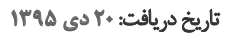

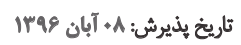

كليدوازوها:

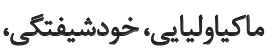
شخصيت ضيلاجتماعي، اختنالات شخصيت

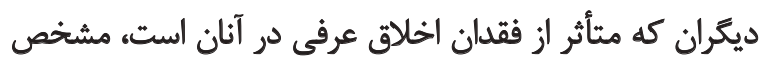
dales

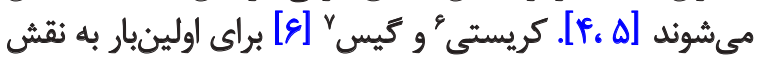

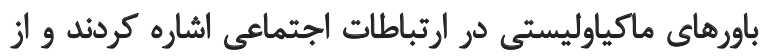

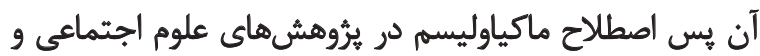
روانشناسى به كار ترفته شده است.

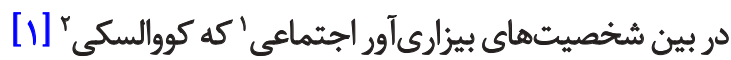

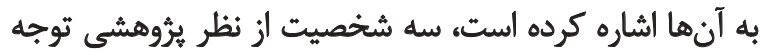

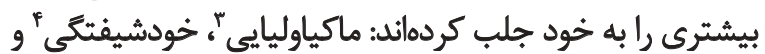

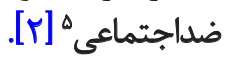
كريستى و كيس با اين اعتقاد كه باورهاى افراده شالوده تعاملات

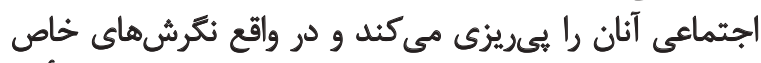

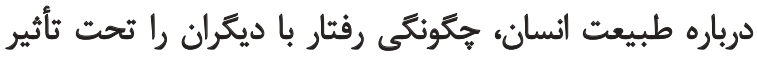

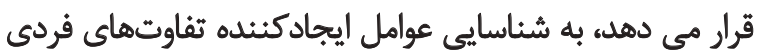

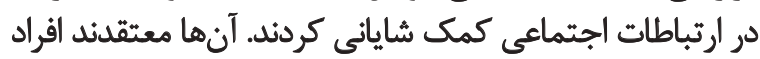

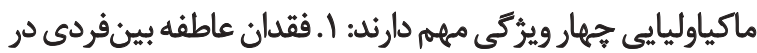

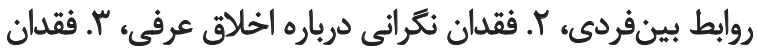
"رُخصيت ماكياوليايى" مفهومى است كه در سالهاي اخير،

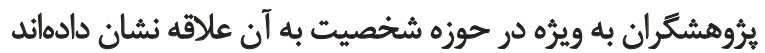

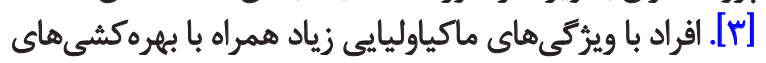

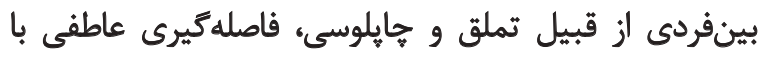


به دست مى آيند. آيزنك براى آشكار كردن صفات شخصيت از

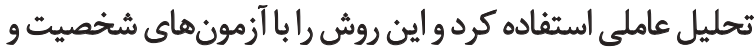

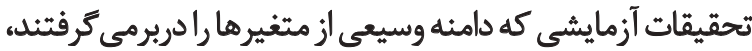

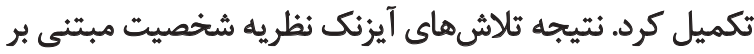

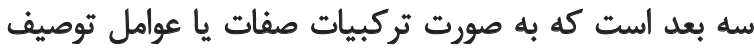

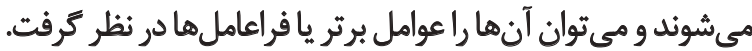

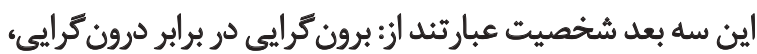

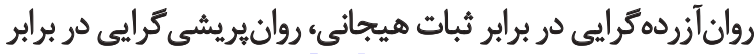
كنترل تكانه (يا عملكرد فراخود) [19]

مرورى بر ادبيات يُوهشى مشخص ميى كند كه همبستههاى

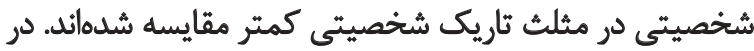

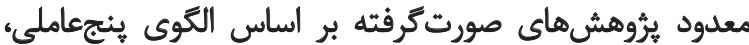

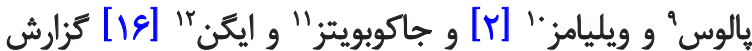

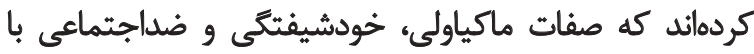

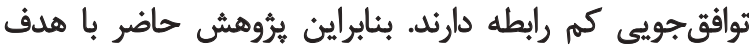

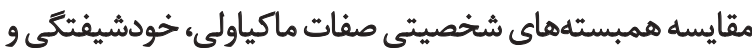

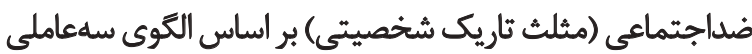

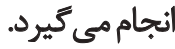

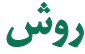
يُروهش حاضر از نوع توصيفى همبستخى است. جامعه آمارى

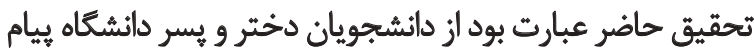

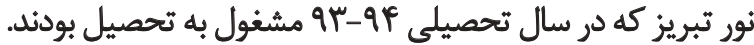

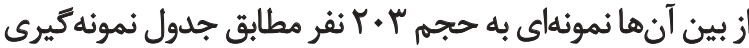

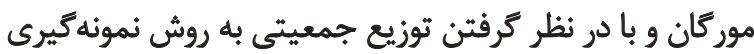

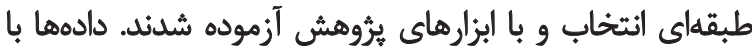

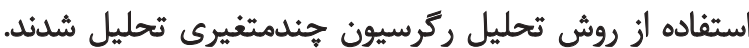

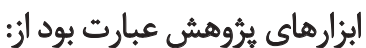

يرسشنامه شخصيث ماكياوليايى (Mach-IV) كريستى و كيس [ع] به منظور سنجش ويزّكى هاى ماكياوليايى

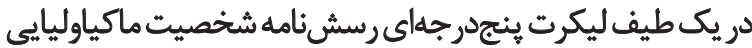

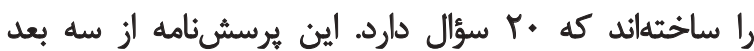

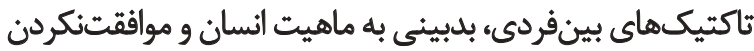

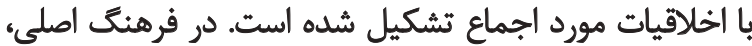

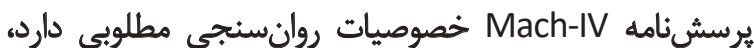

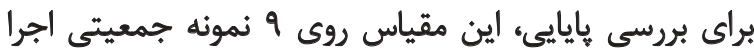

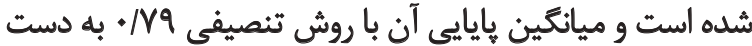

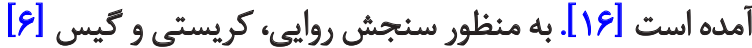

9. Paulhus

10. Williams

11. Jakobwitz

12. Egan
آسيب روانى واضح، f. تعهد ايدئولوريكيى كم [V]].

خودشيفتكى كه يكى از مفاهيم قديمى در روانشناسى به به

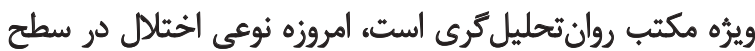

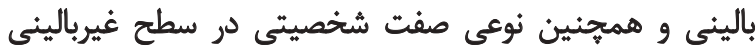

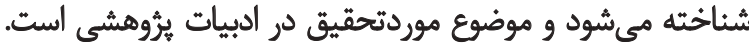

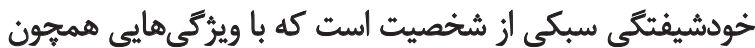

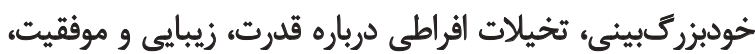

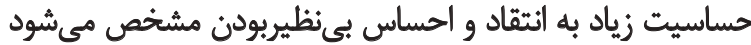

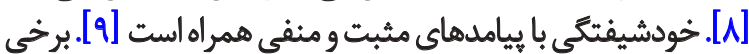

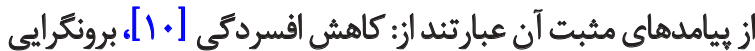

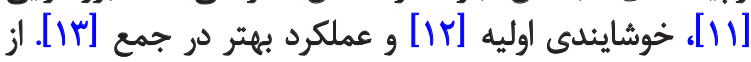

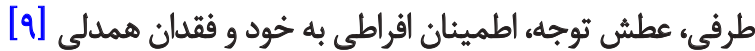

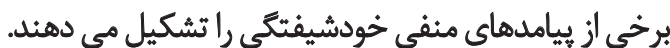

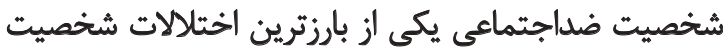

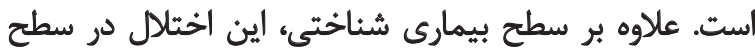

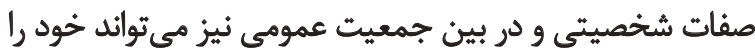

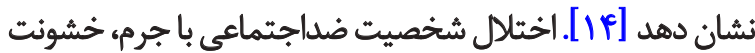

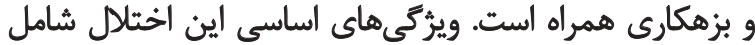

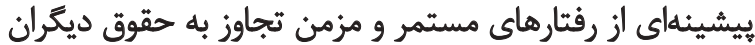

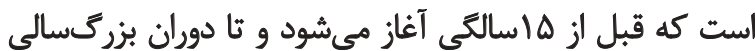

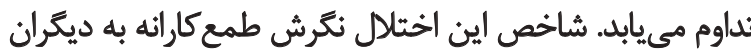

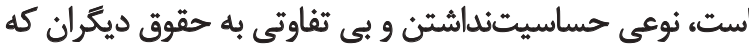

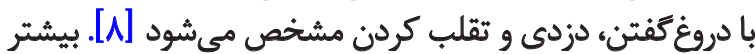

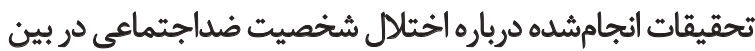

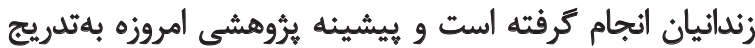

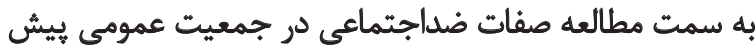

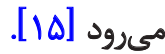

با وجود ريشههاى متفاوت تشكيلدهنده، اين سه شخصيت

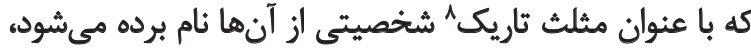

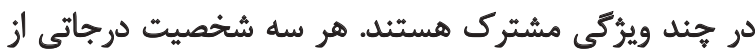

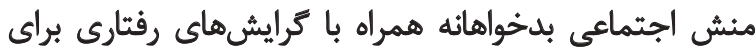

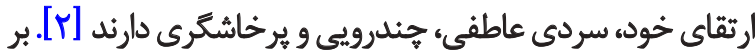

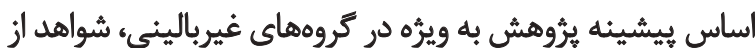

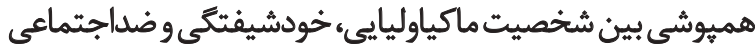

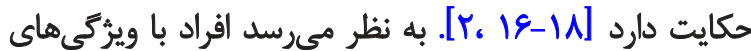

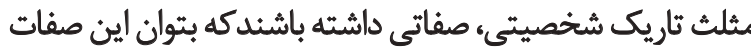

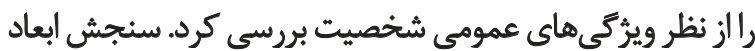

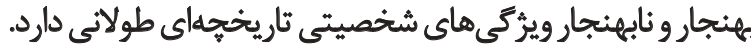

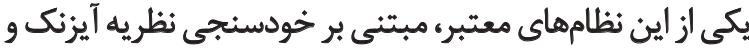

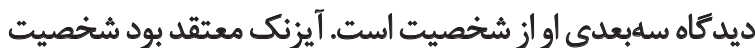

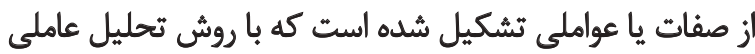




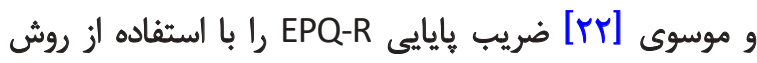

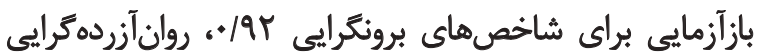

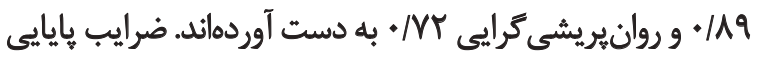

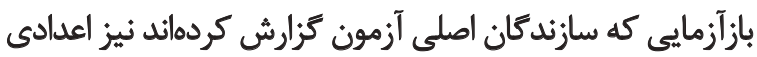

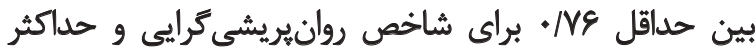

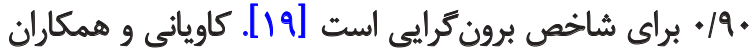

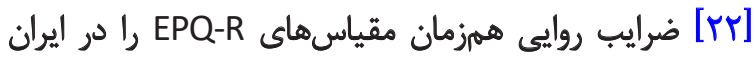

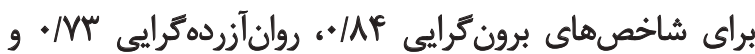

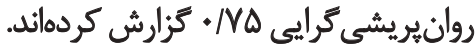

ياقتهها

جمعيت شناختى نمونه تحقيق در جدول شماره ا نشان داده

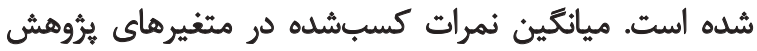

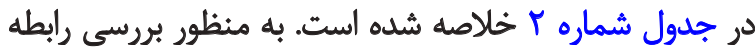

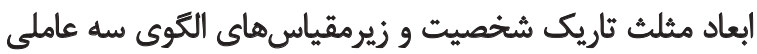

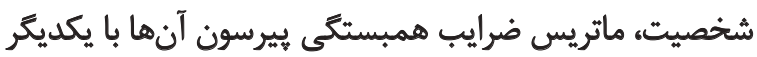

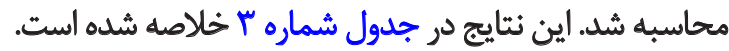
به منظور تعيين دقيقتر رابطه و نيز تشخيص سهرم متغيرهاى

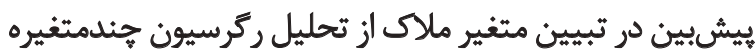

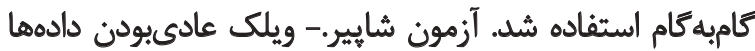

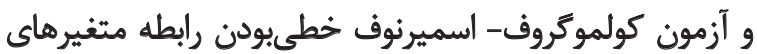

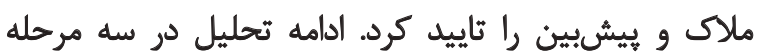

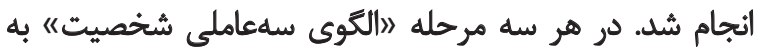
عنوان متغير بيشبين وارد معادله ركرسيون شد. در مرحله الول

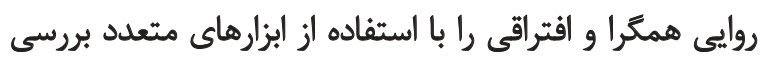

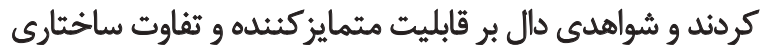

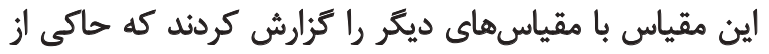

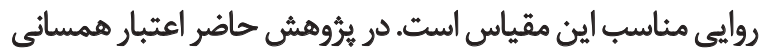

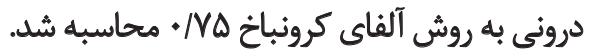

برسش بامه شخصيت هندمحورى باليني ميلون

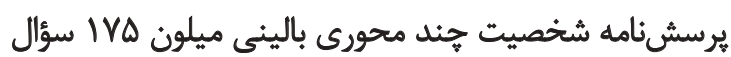

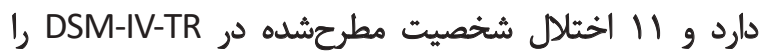

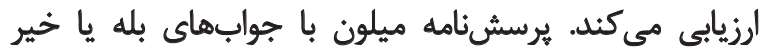

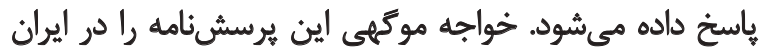

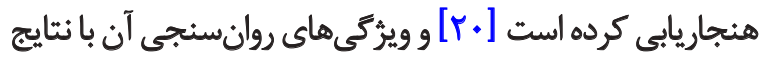

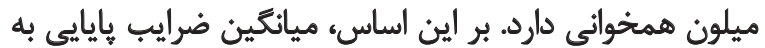

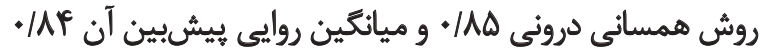

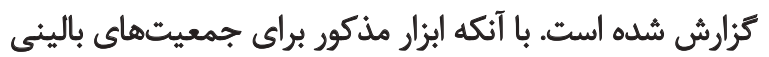

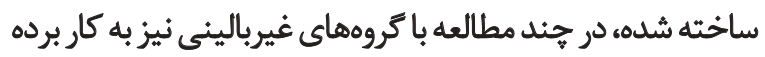

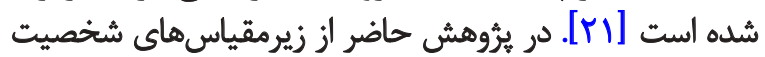

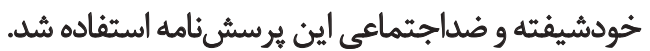

يرسش نامه شخصيثي آيؤنى فرم تجديدنظر شبده (EPQ-R)

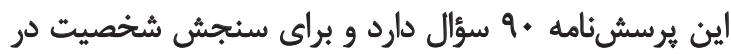

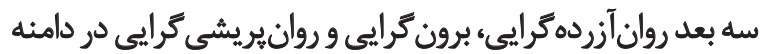

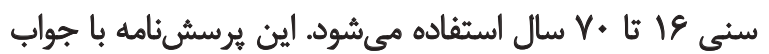

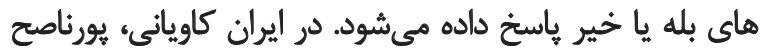

جدول ا. مشخصات جمعيت شئاختى نمونه تحقيق

\begin{tabular}{|c|c|c|c|c|}
\hline انحراف استاندارد & ميانكين سنى & درصد & تعداد & \\
\hline$V / P Q$ & YNIV & VI/F & IPQ & كروه مؤنث \\
\hline V/gr & MVlar & TNE & $\Delta A$ & كروه مذكر \\
\hline V/er & rq. & $1 .$. & $r \cdot r$ & كل نموئه \\
\hline
\end{tabular}

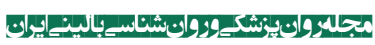

جدول rا. ميانكين نمرات كسب شده و همبستكى ها در متغير هاي يُروهش

\begin{tabular}{|c|c|}
\hline ميانكين (انحراف معيار) & متغير \\
\hline MT/TA(V/N) & شخصيت ماكياوليايي \\
\hline $1 Q / \cdot 1\left(r / \Delta r^{\prime}\right)$ & شخصيت خُودشيفته \\
\hline$V /(Y / A T)$ & شخحصيت ضلاجتماعى \\
\hline $1 . / \mathbb{T}^{e}(\Delta / \mathrm{T})$ & روان آزردهكر ائي \\
\hline $\mid W / \cdot \Psi\left(T / \Delta T^{\prime}\right)$ & برون كرايى \\
\hline$r / \Delta A(T / 19)$ & روانيُيشى كرايى \\
\hline
\end{tabular}

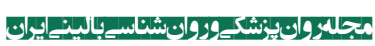


جدول r. ماتريس ضرايب همبستكى بين ابعاد مثلث تاريك شخصيت و زيرمقياس هاى الكَّى سهعاملى

\begin{tabular}{|c|c|c|c|c|c|c|c|}
\hline \multirow[t]{6}{*}{9} & $\Delta$ & $p$ & $r$ & r & 1 & Lod & \\
\hline & & & & & 1 & 1- شخصيت ماكياوليايي & \multirow{3}{*}{ مثلث تاريك شخصيت } \\
\hline & & & & 1 & $.110^{\circ}$ & r- شخصييت خودشيفته & \\
\hline & & & 1 &.$/ 4 T^{\circ}$ &.$/ r V^{* *}$ & "r- شخصيت ضف|جتماعى & \\
\hline & & 1 & . / reses & .111 & . /egen & مأ- روان أزردهذكرايى & \multirow{3}{*}{ الكوى سلهعاملى } \\
\hline & 1 & $\cdot|f|^{* *}$ & . /prese &.$/ 1 \gamma^{*}$ & . MY & ه- روانيريشى كرايع & \\
\hline 1 &.$/ 1 Y$ &.$- / M I^{* *}$ & .1 .8 & $\cdot . / 1 \mathrm{r}$ & $=-\cdot / \pi$ & ع- برون كرايى & \\
\hline
\end{tabular}

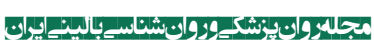

$\mathrm{P}<\cdot 1 \cdot 1 *{ }_{.} \mathrm{P}<+1 \cdot \bullet \Delta^{*}$

جدول F. تحليل ركرسيون متغير شخصيت ماكياوليايى بر الكوى سه عاملى شخصيت

\begin{tabular}{|c|c|c|c|c|c|c|c|c|c|}
\hline Sig. & $\mathbf{t}$ & b & B & Sig. & $\mathbf{F}$ & $\mathbf{R}^{2}$ & $\mathbf{R}$ & مثغير هيشبين & كام \\
\hline .1 .01 & V/er &.$/ 48$ & $.18 \pi$ & $.1 .+1$ & $\Delta \Delta / T Y$ & $\cdot M I$ & .148 & روان آنزرده & اول \\
\hline $\begin{array}{l}.1 .01 \\
.1 .4\end{array}$ & $\begin{array}{l}\Delta / M \\
T / M \Delta\end{array}$ &.$/ 119$ & $\begin{array}{l}.(\Delta)^{\circ} \\
.(\Delta 1)\end{array}$ & $.1 .+1$ & $r v / \cdot \Delta$ & $\cdot / M$ &.$/ 4 A$ & روان أنزدئكرايي & دوم \\
\hline
\end{tabular}

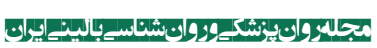

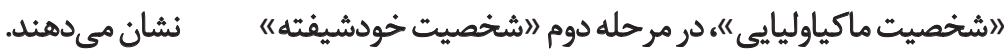

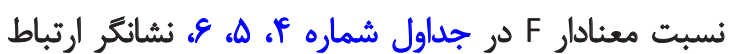

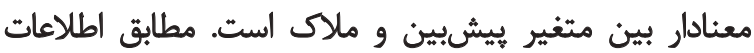

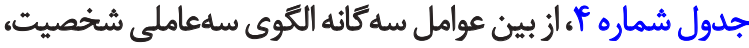

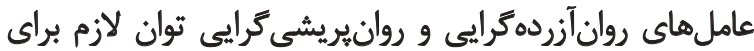

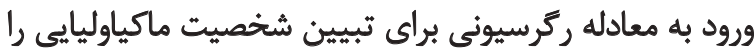

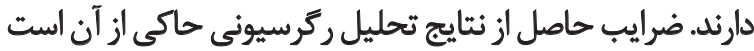

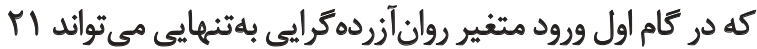

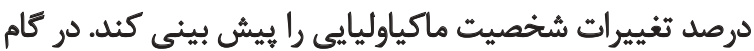

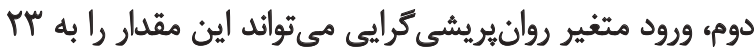
درصد ارثقا دهد. مطابق اطلاعات جدول شماره هـ از بين عوامل سه كائه الكوى

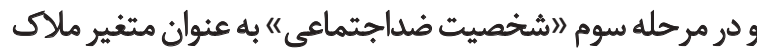
وارد معادله ركرسيون شدمند.

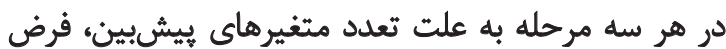

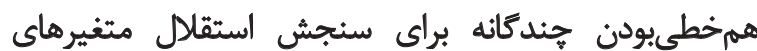

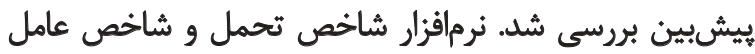

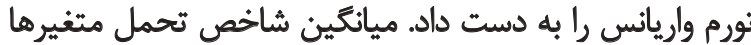

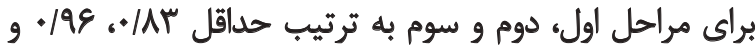

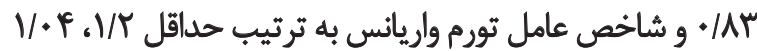

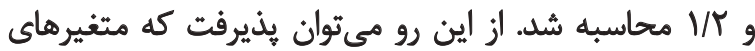

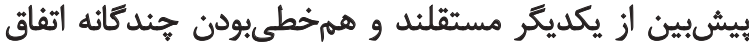

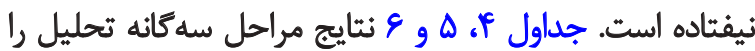

جدول هـ تحليل ركر سيون متغير شخصيت خودشيفته بر التوى سه عاملى شخصيت

\begin{tabular}{|c|c|c|c|c|c|c|c|c|c|}
\hline Sig. & $t$ & $\mathbf{b}$ & B & Sig. & $\mathbf{F}$ & $\mathbf{R}^{2}$ & $\mathbf{R}$ & مثغيو بيشنين & كام \\
\hline .1 .1 & $r / 4 F$ &.$/ N \gamma$ & $\cdot / K A$ & $.1 \cdot 1$ & $\Delta / 9 \gamma$ & $.1+\mu$ &.$/ / V$ & روانيريشى كرايي & اول \\
\hline $\begin{array}{l}. / .+r \\
. \bullet r\end{array}$ & $\begin{array}{l}r / \cdot r \\
\text { r/qY }\end{array}$ & $\begin{array}{l}\cdot /{ }_{1} \\
\cdot /{ }_{1}\end{array}$ & $\begin{array}{l}. / T \Delta \\
. / T I\end{array}$ & $.1 . .1$ & $V / \Delta)$ & $.1 \cdot v$ & $\cdot / r V$ & برونيبريشى برايي & sq \\
\hline
\end{tabular}


جدول 9. تحليل ركرسيون متغير شخصيت ضداجتماعى بر الكوى سه عاملى شخصيت

\begin{tabular}{|c|c|c|c|c|c|c|c|c|c|}
\hline Sig. & $\mathbf{t}$ & $\mathbf{b}$ & B & Sig. & $\mathbf{F}$ & $\mathbf{R}^{\mathbf{2}}$ & $\mathbf{R}$ & متغير ييش بين & تكام \\
\hline $.1 \cdot .1$ & s/DV &.$/ 4 T$ &.$/ 8 A$ & $.1 .+1$ & $T H / T A$ &.$/ M$ &.$/ p t$ & روانيريشى كرايى & اول \\
\hline $\begin{array}{l}.1 \cdot .1 \\
.1 \cdot .1\end{array}$ & $\begin{array}{l}\text { p/pe } \\
\text { p/pe }\end{array}$ &.$/ 4$ &.$/ 4 A$ & $+1+\infty 1$ & $M T / 81$ & $\cdot / K E$ & $\cdot|\Delta|$ & روانيريشى رأنرايى & دوم \\
\hline
\end{tabular}

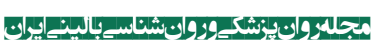

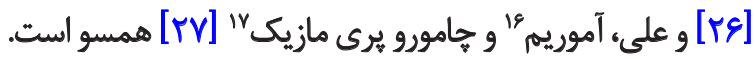

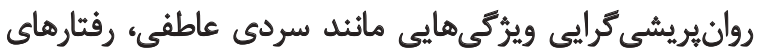

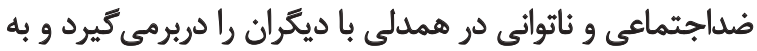

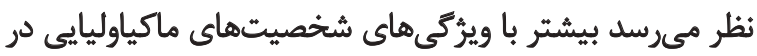

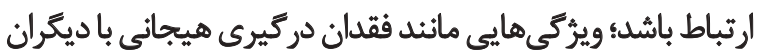

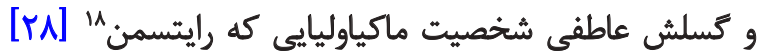

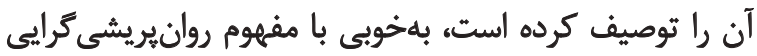

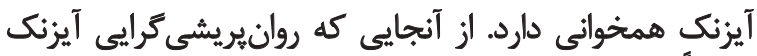

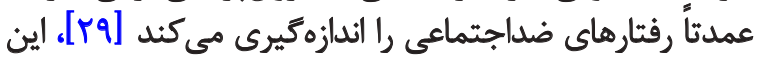

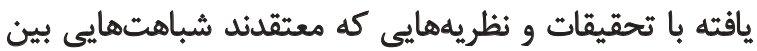

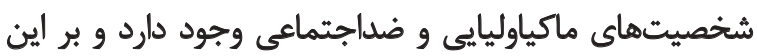

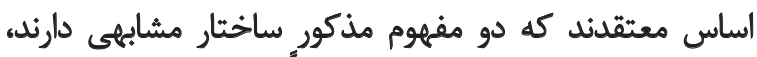

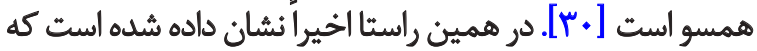

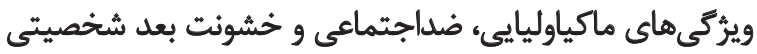

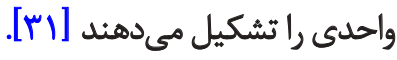

ب- شخصيت خودشيفته با روانيريشى كرايى و برونگرايى

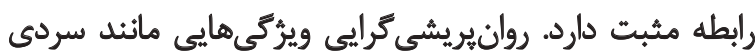

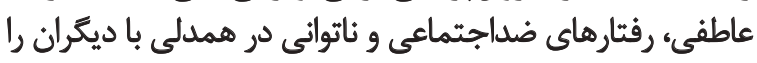

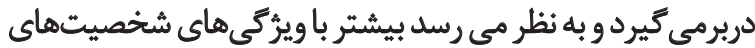

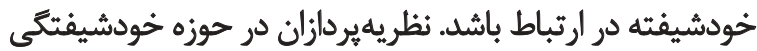

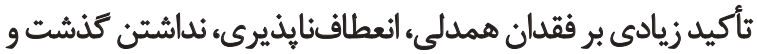

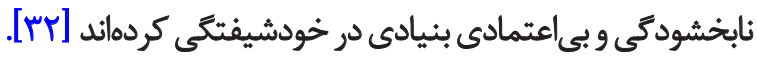

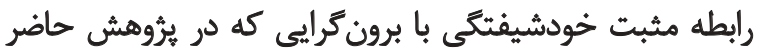

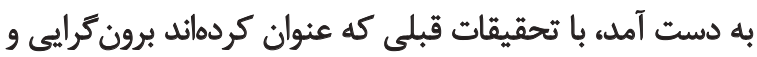

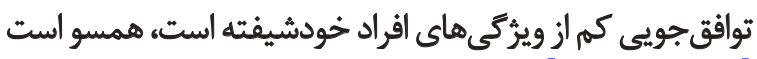

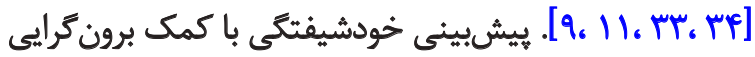

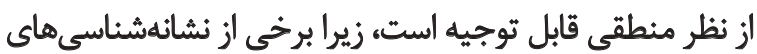

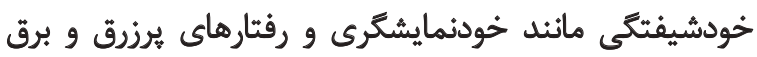

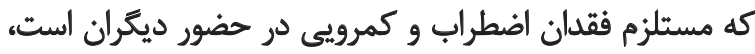

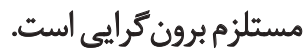

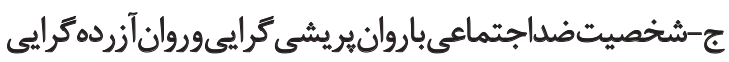

16. Amorim

17. Chamorro-Premuzic

18. Wrightsman

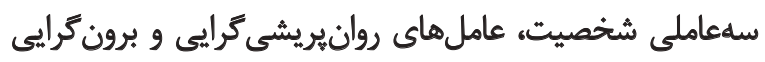

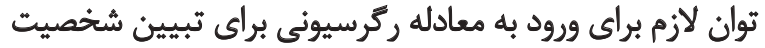

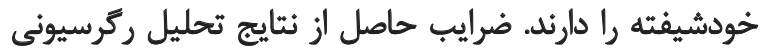

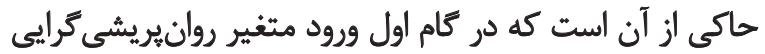

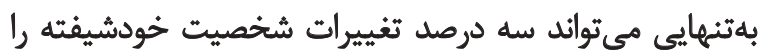

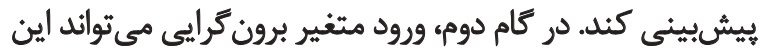
مقدار را به V درصد ارتقا دهد.

مطابق اطلاعات جدول شماره \&ء از بين عوامل سهكانه

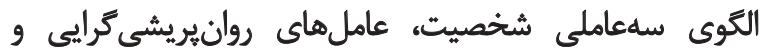

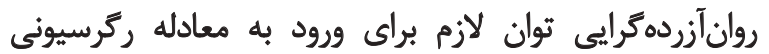

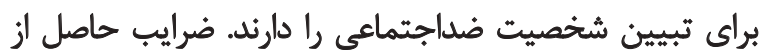

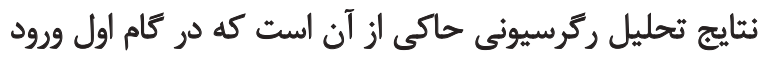

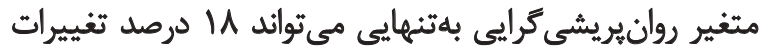

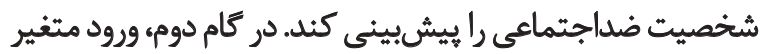

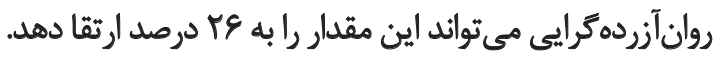

ثִ

يروزهش حاضر با هدف مقايسه همبستهانى شخصيتى صفات

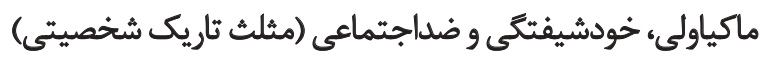

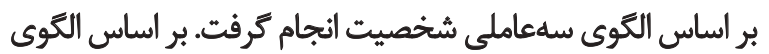
سهعاملى آيزنك اين يافتهها به دست آمدا:

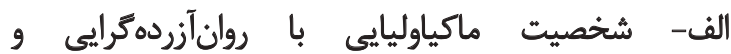

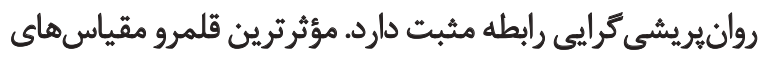

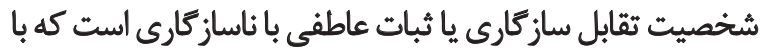

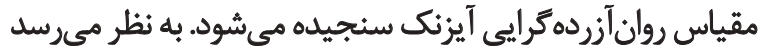

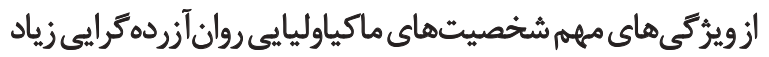

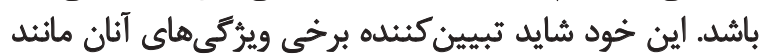

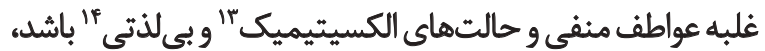

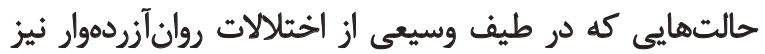

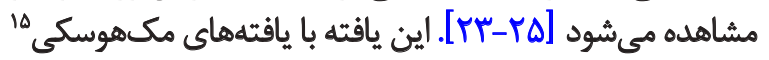

15. McHoskey 
رابطه مثبت دارد. آيزئك، فرد روانيريش را به بهنو عنوان كسى كه

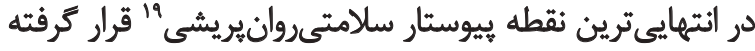

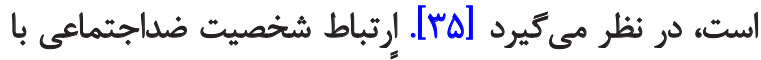
روانيريشى رايى آيزنك كاملاً قابل انتظار است، زير إيرا مقياس

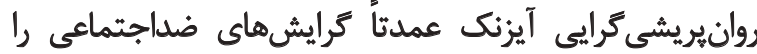

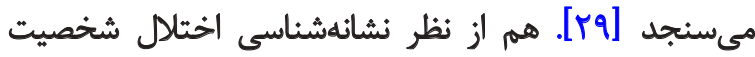

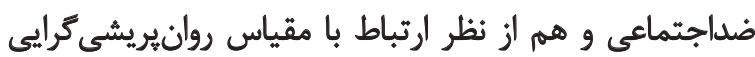

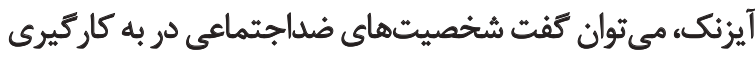

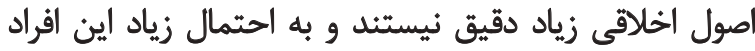

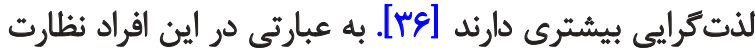

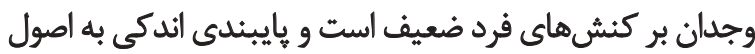

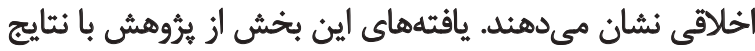

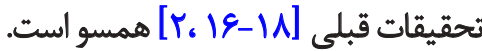

\section{نتيجنيكيرى}

بر اساس نتايج حاصلشده و در جمعبديندى كلى، مئتوان نتيجه تيرى كرد وجه مشترك شخصيتى مطابق القوى سهاعلى

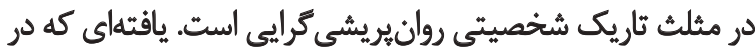

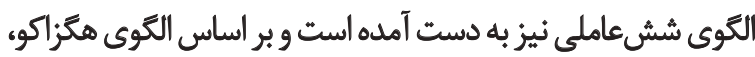

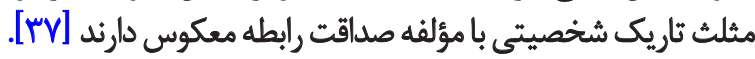
يثروهش حاضر از دو نظر محدوديت داشت: اول اينكه ابزارهاى

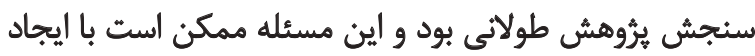

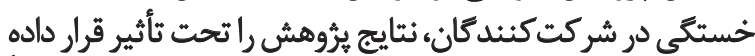

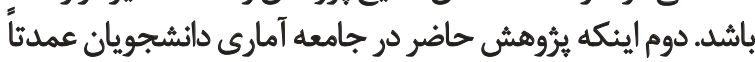

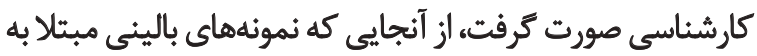

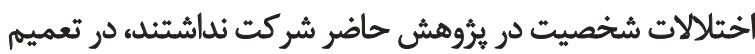

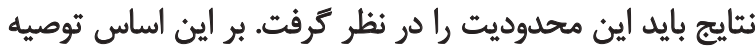

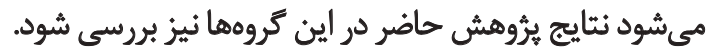

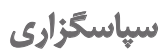

از دانشجويان دانشكاه بيام نور مركز تبريز كه در انجام اين كار

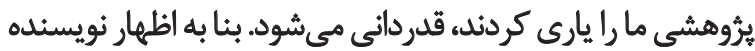

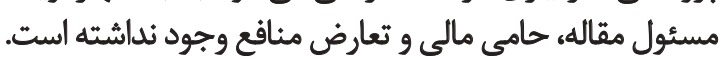




\section{References}

[1] Kowalski RM. Behaving badly: Aversive behaviors in interpersonal relationships. Washington, D. C.: American Psychological Association; 2000.

[2] Paulhus DL, Williams KM. The dark triad of personality: Narcissism, Machiavellianism, and psychopathy. Journal of Research in Personality. 2002; 36(6):556-63. doi: 10.1016/s0092-6566(02)005056

[3] Rauthmann JF. Towards multifaceted Machiavellianism: Content, factorial, and construct validity of a German Machiavellianism Scale. Personality and Individual Differences. 2012; 52(3):34551. doi: 10.1016/j.paid.2011.10.038

[4] Kerekes Z. The Presence of others, prosocial traits, Machiavellianism. Social Psychology. 2010; 41(4):238-45. doi: 10.1027/18649335/a000032

[5] AlAïn S, Carré A, Fantini-Hauwel C, Baudouin J, Besche-Richard C. What is the emotional core of the multidimensional Machiavellian personality trait? Frontiers in Psychology. 2013; 4:1-8. doi: 10.3389/fpsyg.2013.00454

[6] Christie R. Studies in Machiavellianism. London: Academic Press; 1970.

[7] Christie R. Why machiavelli? In: Christie R, Geis F, editors. Studies in Machiavellianism. New York: Academic Press; 1970.

[8] American Psychiatric Association. Diagnostic and statistical manual of mental disorders. Washington, D. C.: American Psychiatric Association; 2013.

[9] Mark Young S, Pinsky D. Narcissism and celebrity. Journal of Research in Personality. 2006; 40(5):463-71. doi: 10.1016/j. jrp.2006.05.005

[10] Watson PJ, Biderman MD. Narcissistic personality inventory factors, splitting, and self-consciousness. Journal of Personality Assessment. 1993; 61(1):41-57. doi: 10.1207/s15327752jpa6101_4

[11] Bradlee PM, Emmons RA. Locating narcissism within the interpersonal circumplex and the five-factor model. Personality and Individual Differences. 1992; 13(7):821-30. doi: 10.1016/01918869(92)90056-u

[12] Oltmanns TF, Friedman JN., Fiedler ER, Turkheimer E. Perceptions of people with personality disorders based on thin slices of behavior. Journal of Research in Personality. 2004; 38(3):216-29. doi: 10.1016/s0092-6566(03)00066-7

[13] Wallace HM, Baumeister RF. The performance of narcissists rises and falls with perceived opportunity for glory. Journal of Personality and Social Psychology. 2002; 82(5):819-34. doi: 10.1037/0022-3514.82.5.819

[14] Board BJ, Fritzon K. Disordered personalities at work. Psychology, Crime \& Law. 2005; 11(1):17-32. doi: 10.1080/10683160310001634304

[15] Ross SR, Lutz CJ, Bailley SE. Psychopathy and the five factor model in a noninstitutionalized sample: A domain and facet level analysis. Journal of Psychopathology and Behavioral Assessment. 2004; 26(4):213-23. doi: 10.1023/b:joba.0000045337.48535.a5

[16] Jakobwitz S, Egan V. The dark triad and normal personality traits. Personality and Individual Differences. 2006; 40(2):331-9. doi: 10.1016/j.paid.2005.07.006
[17] Lee K, Ashton MC. Psychopathy, Machiavellianism, and narcissism in the five-factor model and the HEXACO model of personality structure. Personality and Individual Differences. 2005; 38(7):1571-82. doi: 10.1016/j.paid.2004.09.016

[18] Veselka L, Schermer JA, Martin RA, Vernon PA. Relations between humor styles and the Dark Triad traits of personality. Personality and Individual Differences. 2010; 48(6):772-4. doi: 10.1016/j.paid.2010.01.017

[19] Eysenck HJ, Eysenck SBG. Manual of the eysenck personality questionnaire. London: Hoddler \& Stoughton; 1975.

[20] KhajeMogehi N. [Development and validation of Millon Clinical Multiaxial Inventory-II (MCMI-II) (Persian)] [MSc. Thesis]. Tehran: Iran university of Medical science; 1995.

[21] Choca JP, Vandenberg E, Shanley LA. Interpretive guide to Millon Clinical Multiaxiol Inventory. Washington D. C.: American Psychiatric Association; 1997:

[22] Kaviani H, Pournasseh M, Mousavi A. [Standardization and validation of the revised Eysenck personality questionnaire in the Iranian population (Persian)]. Iranian Journal of Psychiatry and Clinical Psychology. 2005; 11(3):304-11.

[23] Costa PT, McCrae RR. Revised NEO Personality Inventory (NEO PI-R) and NEO Five- Factor Inventory (NEO-FFI) professional manual. Odessa, Florida: Psychological Assessment; 1992.

[24] Mennin DS, McLaughlin KA, Flanagan TJ. Emotion regulation deficits in generalized anxiety disorder, social anxiety disorder, and their co-occurrence. Journal of Anxiety Disorders. 2009; 23(7):866-71. doi: 10.1016/j.janxdis.2009.04.006

[25] Demenescu LR, Kortekaas R, den Boer JA, Aleman A. Impaired attribution of emotion to facial expressions in anxiety and major depression. PLoS ONE. 2010; 5(12):e15058. doi: 10.1371/ journal.pone.0015058

[26] McHoskey JW. Machiavellianism and personality dysfunction. Personality and Individual Differences. 2001; 31(5):791-8. doi: 10.1016/s0191-8869(00)00187-2

[27] Ali F, Amorim IS, Chamorro-Premuzic T. Empathy deficits and trait emotional intelligence in psychopathy and Machiavellianism. Personality and Individual Differences. 2009; 47(7):758-62. doi: 10.1016/j.paid.2009.06.016

[28] Wrightsman LS. Interpersonal trust and attitudes towards human nature. In: Wrightsman L, Shaver P, editor. Measures of Personality and Social Psychological Attitudes. New York: Academic Press; 1991.

[29] Day S, Peters E. The incidence of schizotypy in new religious movements. Personality and Individual Differences. 1999; 27(1):55-67. doi: 10.1016/s0191-8869(98)00218-9

[30] McHoskey JW, Worzel W, Szyarto C. Machiavellianism and psychopathy. Journal of Personality and Social Psychology. 1998; 74(1):192-210. doi: 10.1037/0022-3514.74.1.192

[31] Pailing A, Boon J, Egan V. Personality, the dark triad and violence. Personality and Individual Differences. 2014; 67:81-6. doi: 10.1016/j.paid.2013.11.018

[32] Kernberg OF. Agressivity, narcissism and self-distructivness in the psychotrapeutic relationship: New development in the psychopathology and psychotherapy of severe person-ality disorders. New Haven: Yale University Press; 2004. 
[33] Egan V, McCorkindale C. Narcissism, vanity, personality and mating effort. Personality and Individual Differences. 2007; 43(8):2105-15. doi: 10.1016/j.paid.2007.06.034

[34] Miller JD, Gaughan ET, Pryor LR, Kamen C, Campbell WK. Is research using the Narcissistic Personality Inventory relevant for understanding narcissistic personality disorder? Journal of Research in Personality. 2009; 43(3):482-88. doi: 10.1016/j. jrp.2009.02.001

[35] Goulding A. Schizotypy models in relation to subjective health and paranormal beliefs and experiences. Personality and Individual Differences. 2004; 37(1):157-67. doi: 10.1016/j.paid.2003.08.008

[36] Garousi Farshi M. [The new approaches to personality assessment (Persian)]. Tabriz: Jame'e Pazhooh; 2001.

[37] Lee K, Ashton MC. The dark triad, the big five, and the HEXACO model. Personality and Individual Differences. 2014; 67:2-5. doi: 10.1016/j.paid.2014.01.048 
